\title{
La prothèse complète mandibulaire : stabilité et rétention
}

\section{RÉSUMÉ}

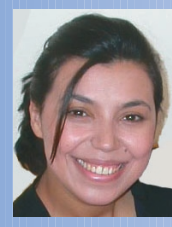

Leila FAJRI

Chirurgien-dentiste,

Spécialiste en prothèse adjointe,

Faculté de médecine dentaire, Rabat,

avenue Allal El Fassi,

rue Mohammed Jazouli,

Madinat Al Irfane, BP 6212,

Rabat-Instituts, Maroc.

Faiza BENFDIL

Professeur assistante en prothèse adjointe.

Bouabid EL MOHTARIM

Professeur de l'enseignement supérieur en prothèse adjointe.

\section{Wafaa EL WADY}

Professeur de l'enseignement supérieur en odontologie chirurgicale.

\section{Ahmed ABDEDINE}

Professeur de l'enseignement supérieur en prothèse adjointe

et en occlusodontie.
Les problèmes de rétention et d'instabilité constituent la principale doléance en prothèse complète mandibulaire.

L'amélioration de l'équilibre prothétique dépendra d'une part, de l'exploitation raisonnée de la surface d'appui, et d'autre part de I'instauration d'un parfait équilibre occlusal.

En fonction de la situation clinique et des conditions économiques des patients, plusieurs modalités thérapeutiques peuvent être envisagées afin d'améliorer la rétention et la stabilité prothétique.

La technique piézographique constitue un excellent recours pour pallier l'instabilité prothétique chez de nombreux patients présentant une importante tonicité musculaire ou une altération du contrôle neuromusculaire.

Dans le souci de majorer l'efficacité masticatoire, la prothèse supraradiculaire est une solution à adopter à chaque fois que le cas le permet. Enfin, avec le développement de l'implantologie, la prothèse supra-implantaire représente une alternative fiable aboutissant à une meilleure intégration prothétique par augmentation de la rétention. Elle améliore de ce fait la qualité de vie des patients édentés complets. 


\section{Introduction}

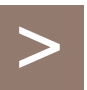

La réalisation d'une prothèse amovible

restaurant un édentement complet mandibulaire est délicate car la stabilité de ce type de prothèse est souvent jugée insuffisante par les patients.

En effet, la préoccupation majeure et la doléance la plus fréquente sont le manque de rétention et de stabilité, ce qui aboutit à une fonction masticatoire limitée et donc à un échec thérapeutique.

L'amélioration des facteurs précités peut se faire soit par l'exploitation raisonnée de la surface d'appui et de l'environnement péri-pro- thétique soit par I'utilisation de moyens de rétention complémentaires.

Ainsi, les solutions thérapeutiques qui permettent de résoudre ce problème d'instabilité prothétique chez un édenté complet mandibulaire sont :

- l'empreinte piézographique ;

- la prothèse complète supra-radiculaire et/ou supra-implantaire associées à des dispositifs de rétention à savoir les attachements.

Toutes ces techniques ont pour objectif d'établir un équilibre prothétique optimal.

\section{Équilibre prothétique}

La réussite de la réhabilitation prothétique se base sur le respect de la triade d'équilibre (fig. 1) dont les différents composants sont:

\section{La rétention}

Elle est définie comme étant la réaction favorable qui s'oppose aux forces exercées sur la prothèse pour l'éloigner de la surface d'appui.
Elle est en rapport avec plusieurs facteurs dont :

\section{$>$ Les facteurs physiques}

- La pesanteur, favorable à la mandibule et défavorable au maxillaire.

- La pression atmosphérique, favorable en cas de joint périphérique effectif.

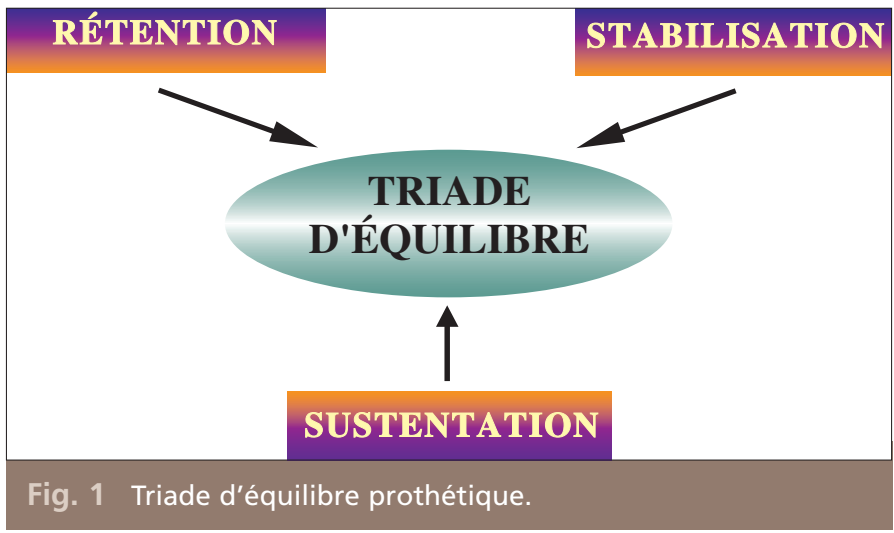


- L'adhésion, qui joue un rôle indirect par la liaison d'un fin film salivaire à la fibromuqueuse d'une part et au matériau des bases d'autre part.

- La viscosité de la salive, qui en augmentant, allonge le temps de décollement de la prothèse de sa surface d'appui.

- L'énergie de surface du matériau de l'intrados prothétique [1].

\section{$>$ Les facteurs physiologiques}

Ils comprennent la qualité de la fibromuqueuse, la qualité de la salive et l'exploitation de la dépressibilité de la muqueuse au niveau du joint périphérique.

\section{$>$ Les facteurs anatomiques}

Ils sont représentés par la profondeur vestibulaire, la largeur et la hauteur des crêtes, la qualité du trigone, la situation de la ligne oblique interne.

\section{La stabilisation}

Elle représente la réaction favorable qui s'oppose aux forces (transversales ou antéroposté- rieures) exercées sur la prothèse parallèlement à la surface d'appui.

Elle est favorisée par la profondeur du vestibule, l'exploitation des ailettes rétro-mylohyoïdiennes et enfin l'établissement d'une occlusion bilatéralement équilibrée qui confère une stabilité supplémentaire et demeure un élément clé de la pérennité de la prothèse totale mandibulaire.

\section{La sustentation}

Elle constitue la réaction favorable qui s'oppose aux forces exercées sur la prothèse perpendiculairement à la surface d'appui. Elle est en rapport avec le support de la prothèse et évite l'enfoncement de la plaque base dans les tissus de soutien lors de la mastication.

Elle dépend à son tour de plusieurs facteurs, à savoir l'étendue de la surface d'appui, la forme des crêtes et enfin la qualité de la fibromuqueuse (une fibromuqueuse abîmée ne peut assurer une bonne sustentation) [2].

\section{Techniques d'amélioration de la rétention et de la stabilité de la prothèse complète mandibulaire}

\section{L'empreinte piézographique}

Selon P. Klein : «la piézographie est le résultat du modelage d'un matériau plastique par la dynamique des organes limitant un espace virtuel ou réel où ce matériau est introduit.» II s'agit donc d'une méthode qui permet d'enregistrer lors de la fonction le jeu des deux grandes masses musculaires circum-prothétiques : la langue et la sangle buccinato-labiale (fig. 2).

Elle permet par conséquent de reproduire en trois dimensions l'espace prothétique mandibulaire de l'édenté complet en intégrant la fonction des éléments anatomiques en rapport avec l'extrados des prothèses [3]. 


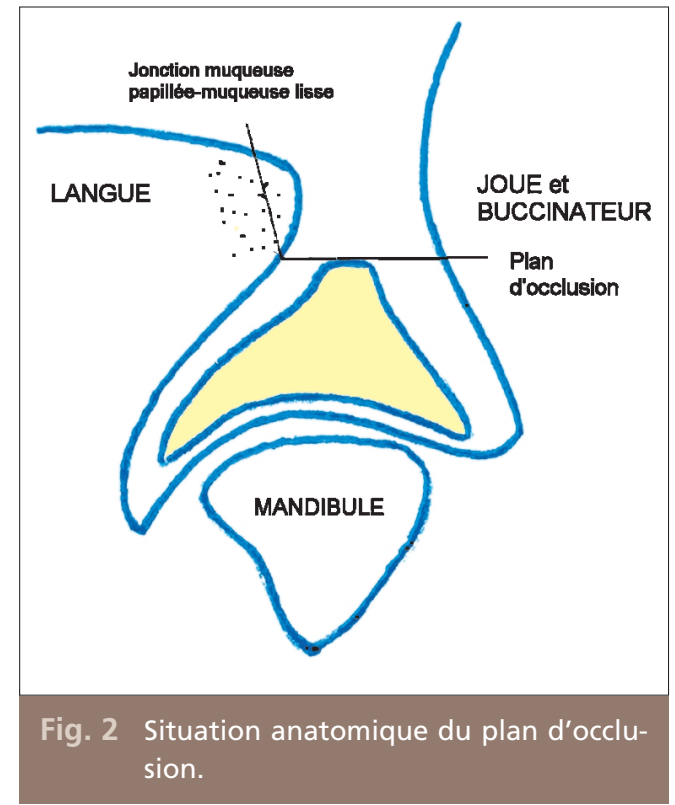

\section{$>$ Indications}

- Les cas de résorptions osseuses importantes [classe III d'Atwood (crêtes plates) ou classe IV (crêtes négatives)].

- Les patients restés très longtemps sans prothèse et ayant acquis de nouvelles habitudes et de nouvelles positions des muscles qui, avec l'ancienneté de l'édentation ne peuvent être restaurées par les méthodes classiques [4].

\section{> Techniques}

Parmi les différentes techniques, la phonation très utilisée par P. Klein nous semble la fonction la plus intéressante comme vecteur de modelage piézographique. Elle présente de nombreux avantages vu que c'est la fonction la plus développée, la plus apte à disjoindre la prothèse de son support muqueux et la moins affectée par la perte des dents. Elle est également l'activité la plus génératrice de forces horizontales actives sur les structures prothétiques. À l'inverse de la mastication et de la déglutition, elle s'effectue dans une cavité non close et en l'absence de contacts occlu-

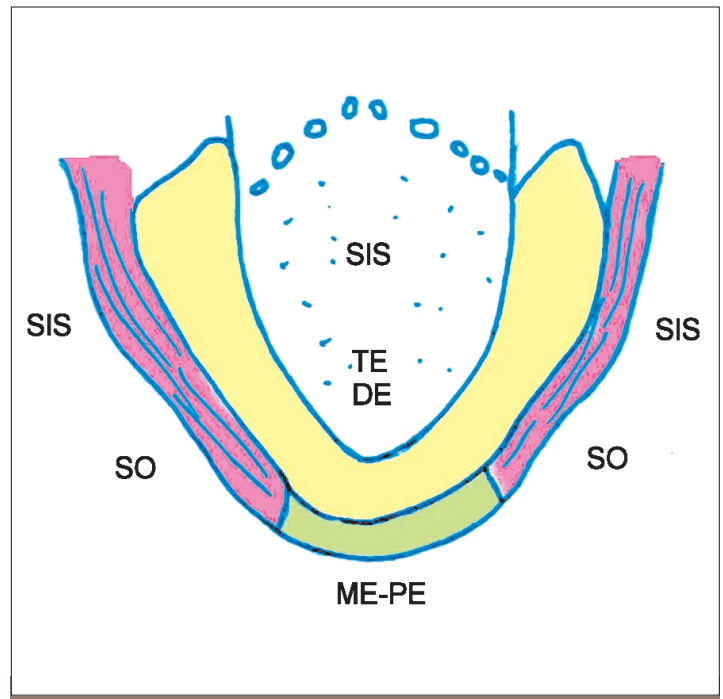

Fig. 3 Phonèmes correspondant à chaque secteur.

saux stabilisants, ce qui permet au matériau d'enregistrement de s'échapper de la cavité buccale.

Elle est le résultat de l'activité synergique des organes périprothétiques, autorisant ainsi le libre jeu du système musculaire bucco-lingual et permettant de raviver la mémoire musculaire souvent aliénée par l'édentement et l'atrophie et de retrouver a priori certaines positions originales de la mandibule sans solliciter le contrôle musculaire volontaire [4-5].

Les matériaux piézographiques les plus utilisés et préconisés par $\mathrm{P}$. Klein sont les résines à prise retard type Fitt de Kerr ${ }^{\circledR}$ ou bien un Thiocol type Permlastic heavy ${ }^{\circledR}$.

L'activation musculaire du modelage en phonation est comme suit (fig. 3 ) :

- pour la région buccinatrice, les phonèmes sont «SIS» répétés six fois: dilatation de la langue et activation du buccinateur, et «SO» une fois dilatation de la langue, contraction de l'orbiculaire et activation du modiolus ;

- pour la région linguale antérieure de la mandibule: l'action centrifuge de la langue est 
obtenue par l'émission du «TE» et «DE» au cours de laquelle une mise en pression de l'air est faite dans la cavité buccale par l'appui de la pointe de la langue sur la face postérieure de la crête antéro-supérieure ; suivie par une brusque décompression par le retrait

\section{Cas clinique $\mathrm{n}^{\circ} 1$}

Une patiente de 63 ans en bon état de santé générale édentée complète, consulte pour une réhabilitation par prothèse complète bimaxillaire. L'interrogatoire a révélé que la patiente n'a jamais été appareillée.

Le choix de la technique piézographique a été dicté par l'importance de la résorption osseuse et l'ancienneté de l'édentement (fig. 4, 5).

Un remarginage des poches de Fish et des régions paralinguales est réalisé sur un porteempreinte individuel (fig. 6), suivi d'un surfaçage avec de I'Impregum ${ }^{\circledR}$ (fig. 7).

Sur le modèle issu de cette empreinte secondaire, une maquette en résine autopolymérisable (Formatray ${ }^{\circledR}$ ) est confectionnée avec un bourrelet en lame de couteau, parfaitement centré sur l'arcade (fig. 8).

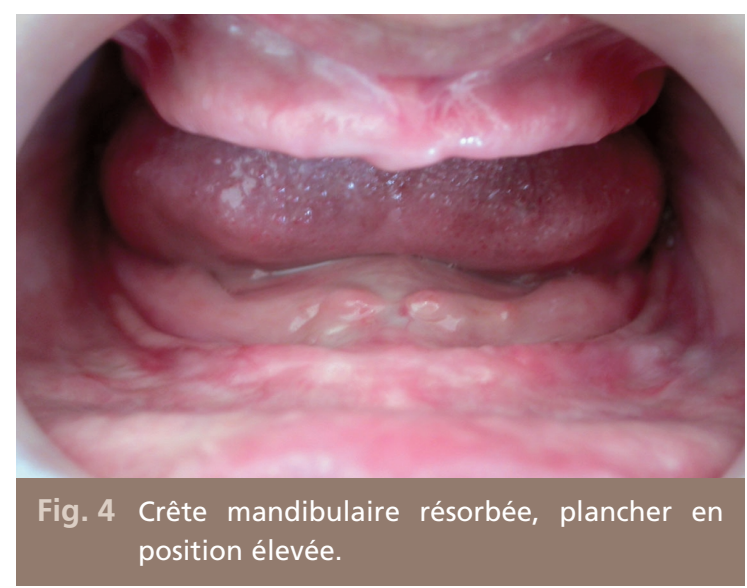

de cette pointe et abaissement de la mandibule. L'action centripète provient des lèvres au moment de l'émission du «ME» et «PE» qui viennent balayer la zone vestibulolabiale pour modeler la région antérieure externe [4-7].

Un polysulfure de moyenne viscosité (Permlastic ${ }^{\circledR}$ ) est préparé et enduit sur les zones à définir. La maquette est alors insérée en bouche. Le modelage s'effectue par mise en œuvre de la phonation et aboutit à une empreinte fonctionnelle de l'extrados prothétique (fig. 9, 10).

Des clés en silicone lourd sont réalisées en vestibulaire et lingual de l'enregistrement de l'extrados, délimitant ainsi le couloir prothétique (fig. 11) où seront montées les dents.

Les bords incisifs et les pointes cuspidiennes des dents prothétiques doivent être alignés sur le plan d'occlusion déterminé par la piézographie. Le groupe postérieur nécessite souvent une réduction dans le sens vestibulo-lingual $[5,7,8]$.

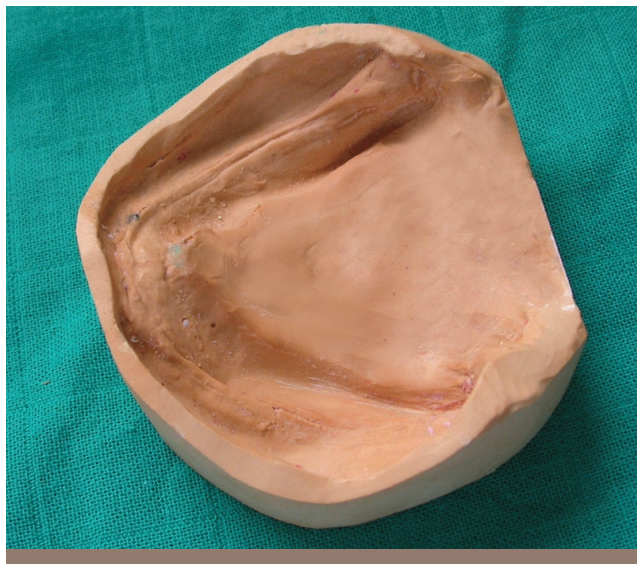

Fig. 5 Modèle issu de l'empreinte primaire. 

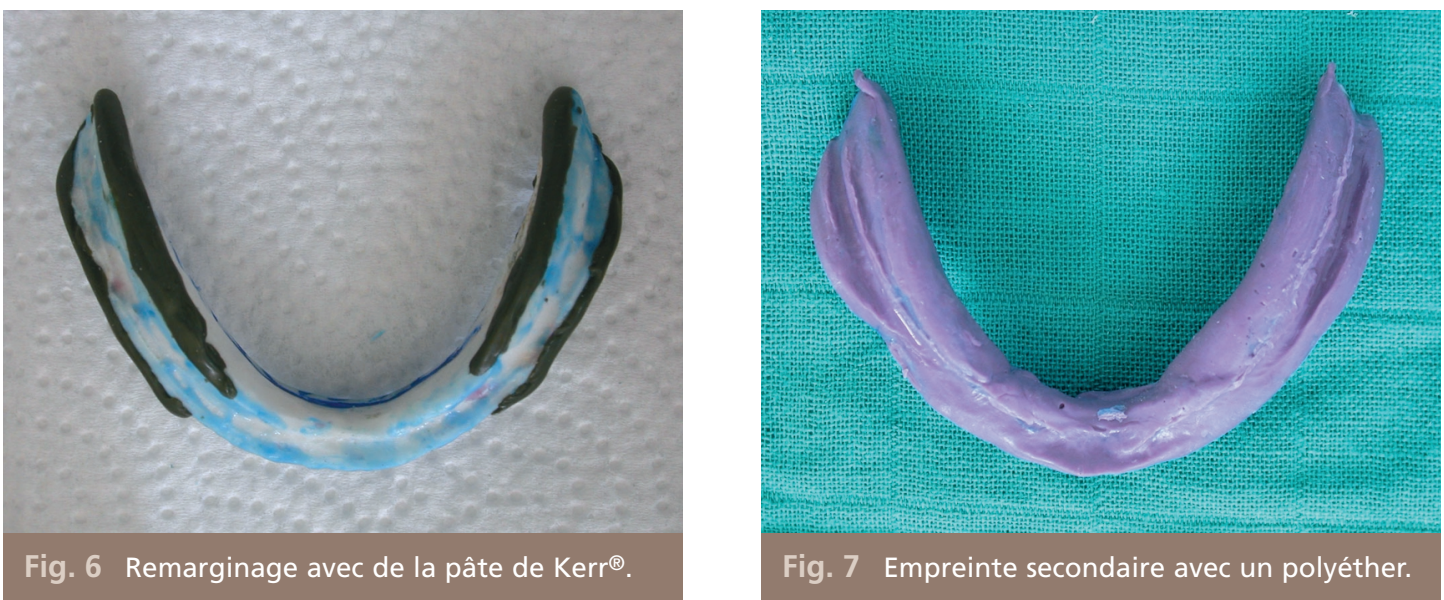

Fig. 7 Empreinte secondaire avec un polyéther.

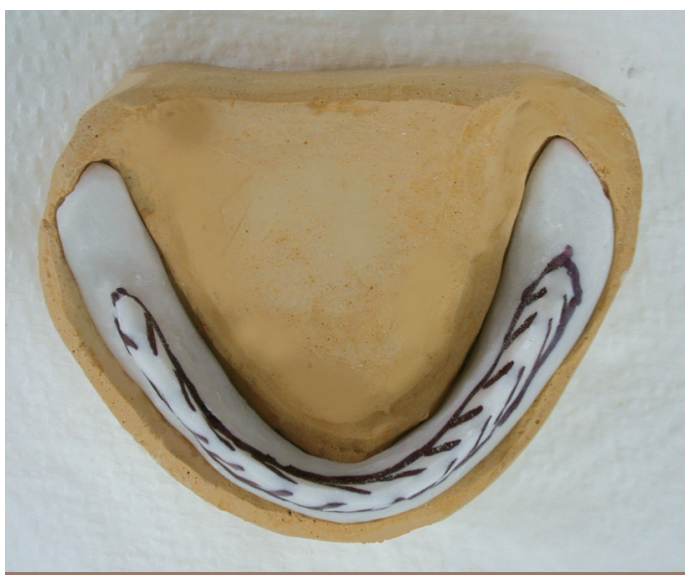

Fig. 8 Maquette en résine.

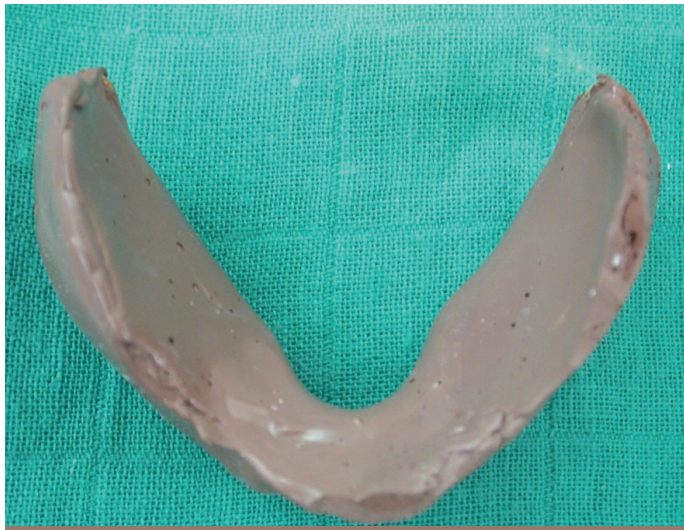

Fig. 9 Empreinte de l'extrados après prononciation des phonèmes.

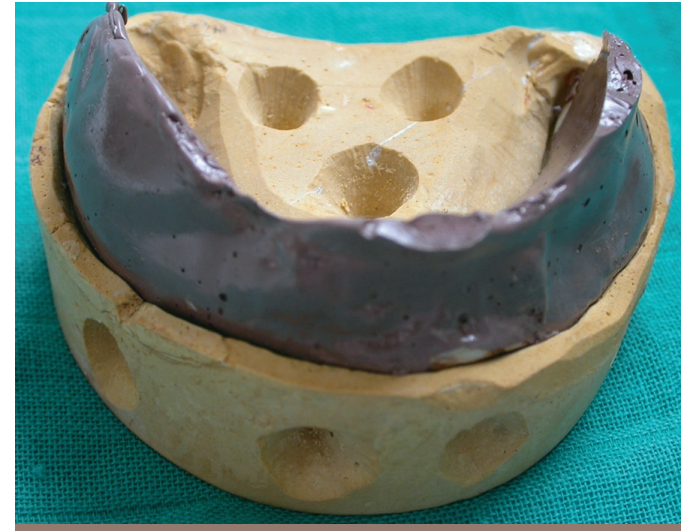

Fig. 10 Réalisation des rétentions pour le repositionnement précis des clés sur le modèle. 


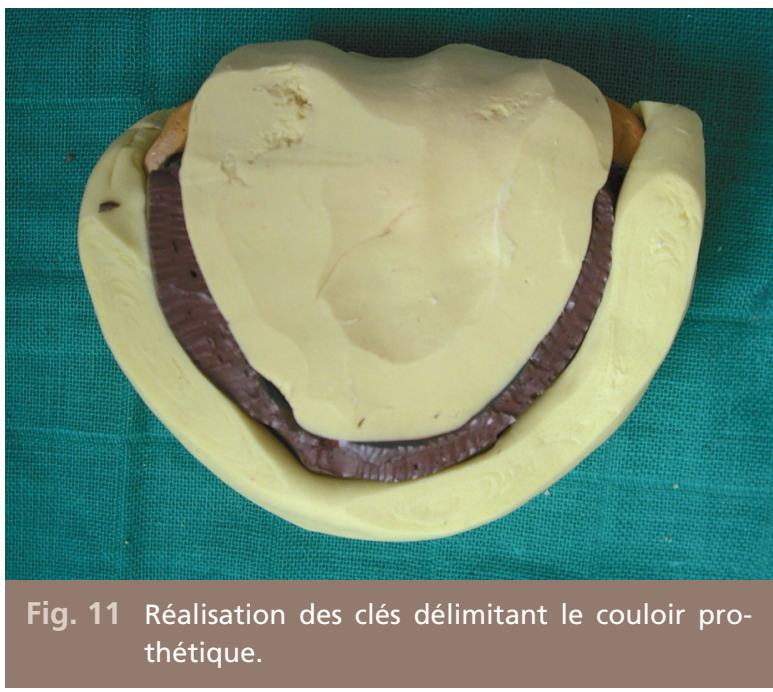

Ceci a permis de majorer la stabilité de la prothèse mandibulaire par l'intégration de la fonction des éléments anatomiques en rapport avec les prothèses pour la définition de la forme de l'extrados.

\section{La prothèse supra-radiculaire avec moyens d'ancrage}

Dans le souci d'obtenir une rétention maximale de nos prothèses, et lorsque des racines résiduelles de bonne valeur prothétique persistent, le praticien peut les utiliser comme support de moyens de rétention complémentaires par le biais d'une prothèse supra-radiculaire.

\section{$>$ Avantages}

La prothèse supra-radiculaire permet :

- le maintien de la proprioception desmodontale: les patients utilisant des prothèses supra-radiculaires conservent une efficacité masticatoire allant jusqu'à $90 \%$. Cela s'explique par le rôle de la proprioception dans la programmation du cycle masticatoire et dans l'activité des masséters. La coordination neuromusculaire est alors plus efficace ;

- la stimulation de la salivation [9];

- la conservation du capital osseux par la conservation de l'os alvéolaire, qui constitue un rempart ou relief vertical permettant d'accroître la stabilité ;

- l'augmentation de la rétention par le biais $d$ 'attachements lorsque la valeur intrinsèque des racines résiduelles le permet [10].

\section{$>$ Conditions cliniques}

Plusieurs impératifs sont nécessaires à la réalisation de la prothèse supra-radiculaire.

L'environnement parodontal doit être sain :

- la présence d'une barrière de gencive attachée est indispensable ;

- la hauteur de l'os résiduel doit être suffisante ;

- les racines ne doivent présenter aucune mobilité avant leur mise en charge.

Absence de forte contre dépouille. Les axes radiculaires doivent être favorables à l'intégration d'un système mécanique [10]. 


\section{> Préparation préliminaire}

Un traitement préalable s'avère souvent indispensable. II s'agit de réaliser :

- des traitements endodontiques ;

- un traitement parodontal : ce dernier constitue une étape importante de la préparation [11].

\section{> Les systèmes de rétention}

Le dispositif de rétention doit répondre à certains critères généraux :

- simplicité de la conception : en permettant une compréhension rapide de son fonctionnement et une maintenance aisée aussi bien pour le praticien que pour le patient ;

- rétention suffisante: d'après la littérature une force de rétention de 10 à $20 \mathrm{~N}$ est nécessaire ;

- fiabilité : I'usure se traduit par une diminution de la valeur rétentive. Elle est compensée par activation ou changement des anneaux de rétention [12].

La faible rétention prodiguée par les attachements magnétiques et l'apparition des phénomènes de corrosion intrabuccale conduisent à éviter ce type d'attachement [13].

On distingue deux types d'attachements, unitaire (axiaux) ou barre d'ancrage.

\section{> Les attachements axiaux}

Type bouton-pression, ils comprennent une pièce mâle (patrice) venant s'emboîter dans une pièce femelle (matrice). La partie mâle de l'attachement peut être en résine calcinable permettant une coulée monolithique de l'attachement et de la coiffe, ou bien métallique solidarisée secondairement à la coiffe [14, 15].

La partie femelle est en général solidaire de la prothèse amovible, elle est incluse dans la résine de la base soit au laboratoire soit directement en bouche [16].

La rétention est obtenue par emboîtement des pièces mâles et femelles qui peut être à frottement plus ou moins fort [17].
La force de rétention ne doit pas être excessive afin d'éviter qu'elle soit une source de difficulté de désinsertion pour les patients et qu'elle altère le parodonte des racines supports [16].

\section{> Les barres d'ancrage}

Les barres d'ancrage constituent un moyen prophylactique de conservation et de soulagement des tissus parodontaux des dents piliers. La solidarisation des piliers réduit leur sollicitation lors des déplacements prothétiques, tout en conférant une bonne efficacité rétentive [18].

Ce sont les dispositifs les plus stabilisants mais aussi les plus difficiles à mettre en œuvre.

On les préférera aux attachements axiaux toutes les fois que :

- les axes radiculaires sont parallèles ;

- la répartition topographique des piliers est favorable avec une crête édentée antérieure rectiligne [16] ;

- l'espace prothétique disponible est suffisant.

Elles sont fournies avec un espaceur qui est utilisé pendant l'élaboration de la prothèse. Celui-ci est retiré après polymérisation et l'espace libre créé va permettre une résilience partielle du système.

Sur le plan de la maintenance, un espacement entre la muqueuse et la barre est nécessaire mais il peut être soit trop important facilitant l'élimination de la plaque mais entraînant une hauteur du système qui rend difficile la mise en place des dents prothétiques et une probabilité d'apparition de diapneusie à long terme, soit insuffisant induisant alors une réaction inflammatoire de la muqueuse sous-jacente [19] ; cet espace doit donc être suffisant pour faire passer les brossettes interdentaires qui permettront d'assurer l'élimination de la plaque bactérienne. 


\section{Cas clinique $\mathrm{n}^{\circ} 2$ Prothèse supra-radiculaire avec attachements axiaux}

\section{> Démarche clinique}

Une patiente âgée de 61 ans diabétique équilibrée, se présente à la consultation en vue du renouvellement de ses prothèses.

L'examen endobuccal montre une prothèse complète maxillaire ainsi qu'une prothèse mandibulaire en résine utilisant comme moyen de rétention deux crochets métalliques façonnés au niveau des deux canines, ces dernières comportant d'importantes lésions cervicales (fig. 12).

La qualité du parodonte, le niveau osseux favorable ainsi que les conditions financières de la patiente nous ont orienté vers la réalisation d'une prothèse supra-radiculaire. Le choix du type d'attachements a été en faveur des attachements axiaux en raison de la divergence des axes radiculaires ainsi que de la courbure de la crête antérieure.

La préparation des dents supports (fig. 13) a été réalisée en respectant les impératifs suivants :

- préparation radiculaire pour un tenon cylindro-conique occupant les $2 / 3$ de la racine et laissant apicalement 3 à $4 \mathrm{~mm}$ d'obturation radiculaire ;

- préparation périphérique complète à congé large (016) juxta-gingival (type plateau de Richmond) ;

- un entonnoir est réalisé à l'entrée de la préparation canalaire de façon à obtenir une bonne résistance de la pièce prothétique coulée [20].

Une empreinte servant à la confection des chapes radiculaires a été réalisée selon les techniques classiques des empreintes en double mélange des tenons normalisés (fig. 14).

Le laboratoire réalise les chapes à tenon radiculaire de forme parabolique. Les pièces mâles des attachements en plastique calcinable (OT cap de Rhein) sont fixées sur la maquette des chapes selon l'axe d'insertion et l'ensemble a été coulé en une seule pièce (fig. 15).

Les limites cervicales et l'adaptation des chapes ont été contrôlées lors de l'essayage en bouche (fig. 16).

L'empreinte secondaire de la surface d'appui a été réalisée avec un porte-empreinte individuel adapté et rectifié en bouche.

Tout d'abord, un remarginage est réalisé au niveau du joint périphérique (fig. 17), une fois la rétention jugée satisfaisante, une empreinte de surfaçage entraînant les chapes est réalisée à l'aide d'un polysulfure de moyenne viscosité, le patient est invité à mobiliser sa musculature périphérique et linguale dans les mouvements fonctionnels extrêmes, ce qui permet d'enregistrer la position haute du plancher buccal dans les zones postérieures et des retours fonctionnels affinés dans la zone du joint sublingual (fig. 18).

Les étapes classiques de la prothèse adjointe complète sont réalisées à savoir : 
- la détermination de la dimension verticale d'occlusion ;

- l'enregistrement du RIM ;

- le choix et le montage des dents ainsi que l'appréciation de la valeur esthétique et fonctionnelle du montage lors de l'essayage en bouche ;

- l'occlusion est réglée selon le concept d'occlusion bilatéralement équilibrée.

La prothèse est tout d'abord essayée sans les chapes pour vérifier la sustentation, la rétention et la stabilité. Les chapes surmontées des parties mâles sont alors mises en place et leur ajustage est vérifié.
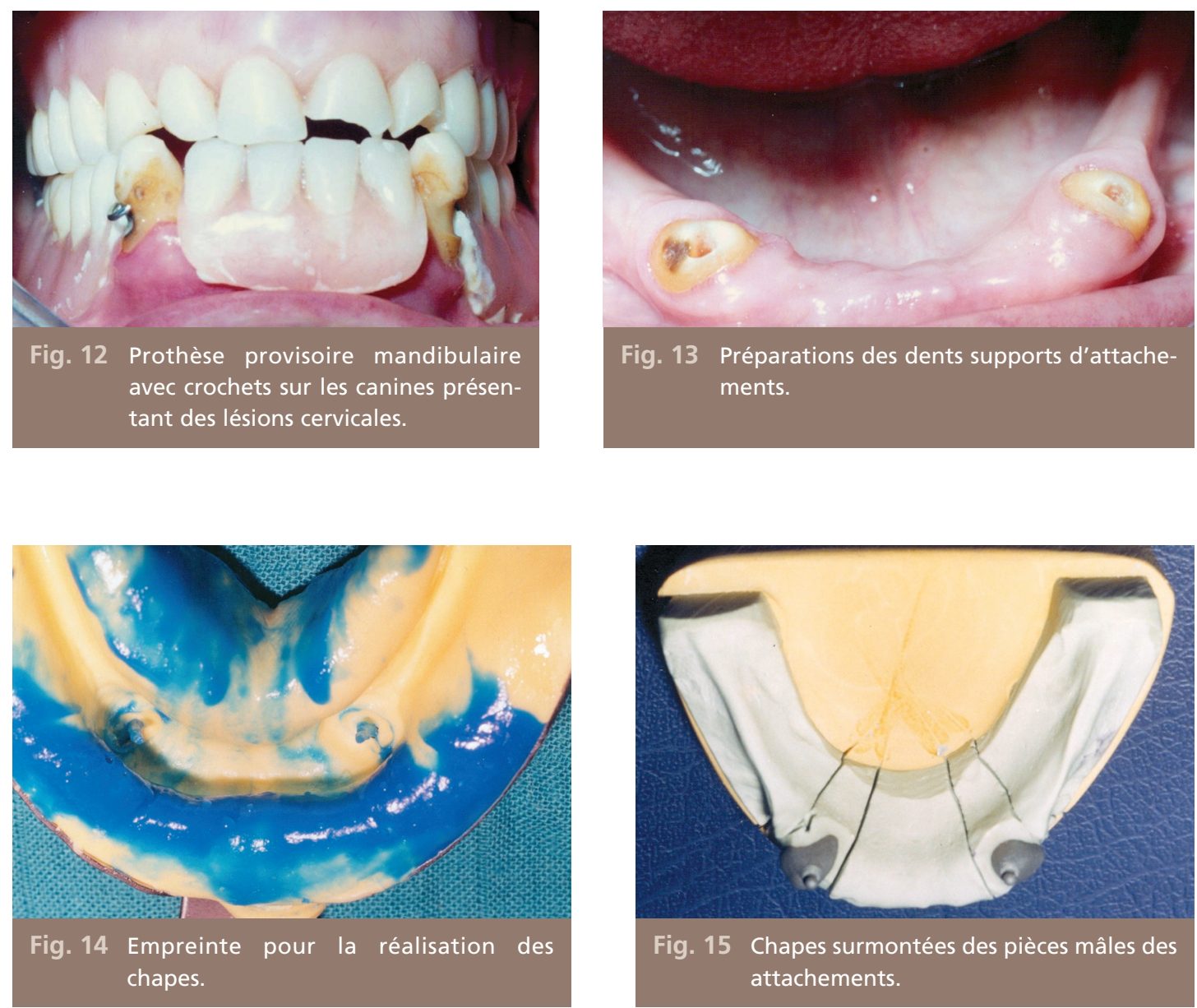

La prothèse mandibulaire est de nouveau essayée, les pièces femelles sont alors placées sur les éléments mâles préalablement espacés (fig. 19).

L'intrados de la prothèse est évidé en regard des chapes (fig. 20) afin de solidariser en bouche et sous pression occlusale la pièce femelle de l'attachement à la prothèse avec de la résine autopolymérisable (fig. 21).

La rétention et la stabilité obtenues au stade de l'empreinte ont été majorées par la mise en place des attachements axiaux supraradiculaires avec un confort accru lors de la mastication rapporté par la patiente pendant les visites de contrôle. 


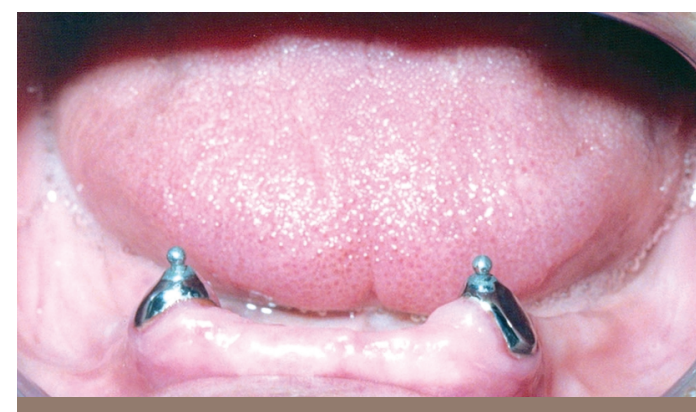

Fig. 16 Chapes essayées en bouche.

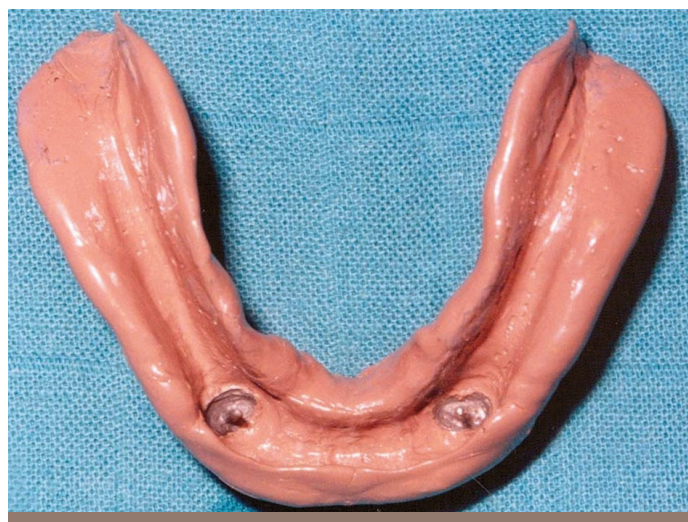

Fig. 18 Empreinte de surfaçage.

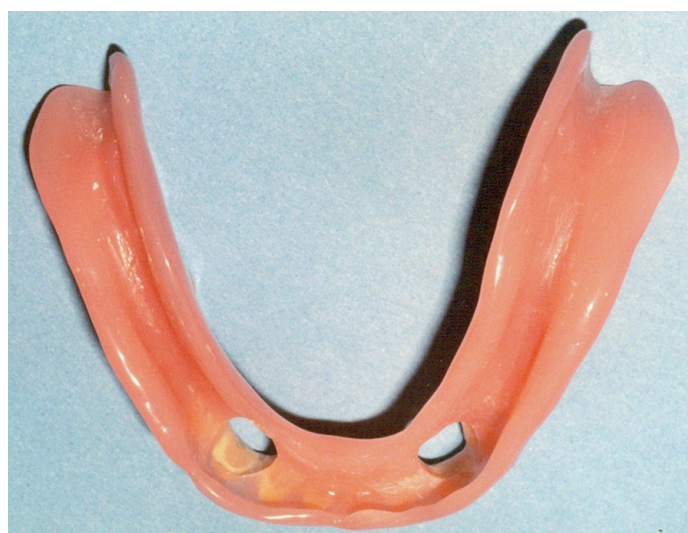

Fig. 20 Évidement de la résine au niveau de l'intrados.

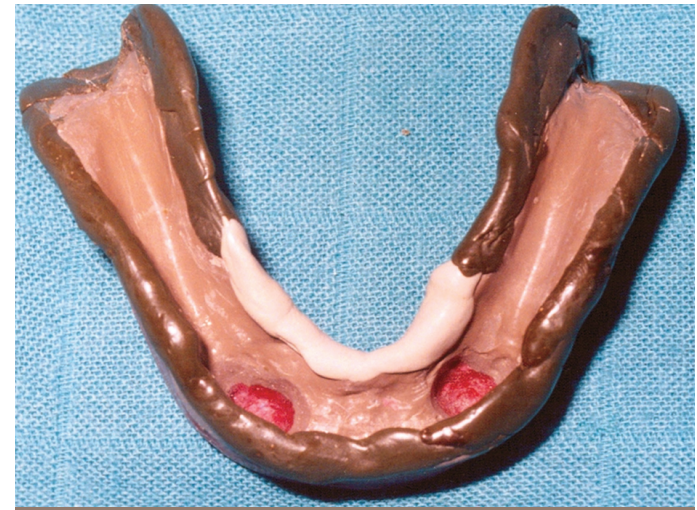

Fig. 17 Enregistrement du joint périphérique.

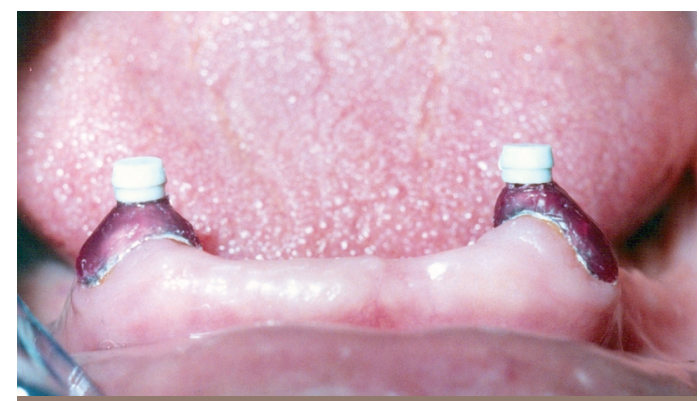

Fig. 19 Mise en place de cire d'espacement avant solidarisation des parties femelles à la prothèse.

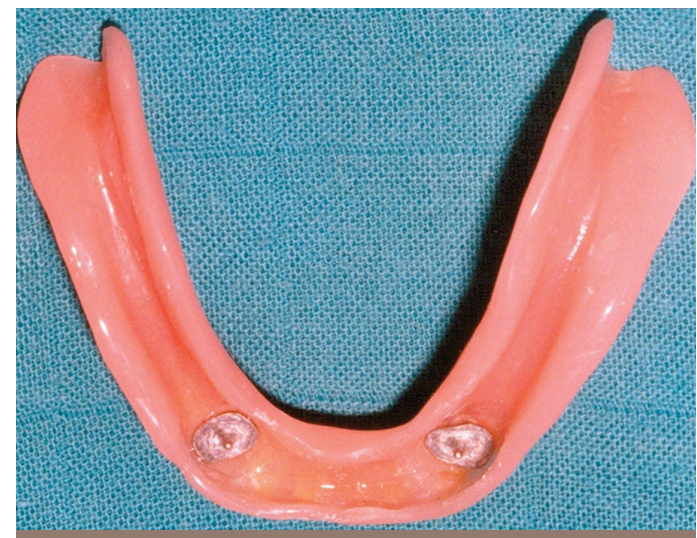

Fig. 21 Vue de l'intrados prothétique (les chapes étant entraînées après solidarisation des parties femelles). 


\section{Cas clinique $n^{\circ} 3$ \\ Prothèse supra-radiculaire avec barre d'ancrage}

\section{$>$ Démarche clinique}

Une patiente âgée de 68 ans s'est présentée pour une réhabilitation par prothèse complète. A l'examen clinique, seules persistaient les deux canines mandibulaires avec un bon rapport couronne clinique - racine clinique et un bon état parodontal.

Les axes radiculaires parallèles et la crête antérieure rectiligne (fig. 22) ont été en faveur de la réalisation d'une barre de conjonction. Les mêmes étapes ont été réalisées, à savoir les préparations (fig. 23) et l'empreinte des chapes objectivant le parallélisme des tenons radiculaires (fig. 24).

La barre d'ancrage a été réalisée en tenant compte de l'emplacement des dents prothétiques antérieures (fig. 25, 26). Elle est essayée puis entraînée dans une empreinte secondaire de la surface d'appui. Après les étapes classiques du rapport intermaxillaire et du montage des dents prothétiques, la barre est scellée en bouche, et le cavalier solidarisé à l'intrados prothétique en bouche avec de la résine autopolymérisable et sous pression occlusale (fig. 27).
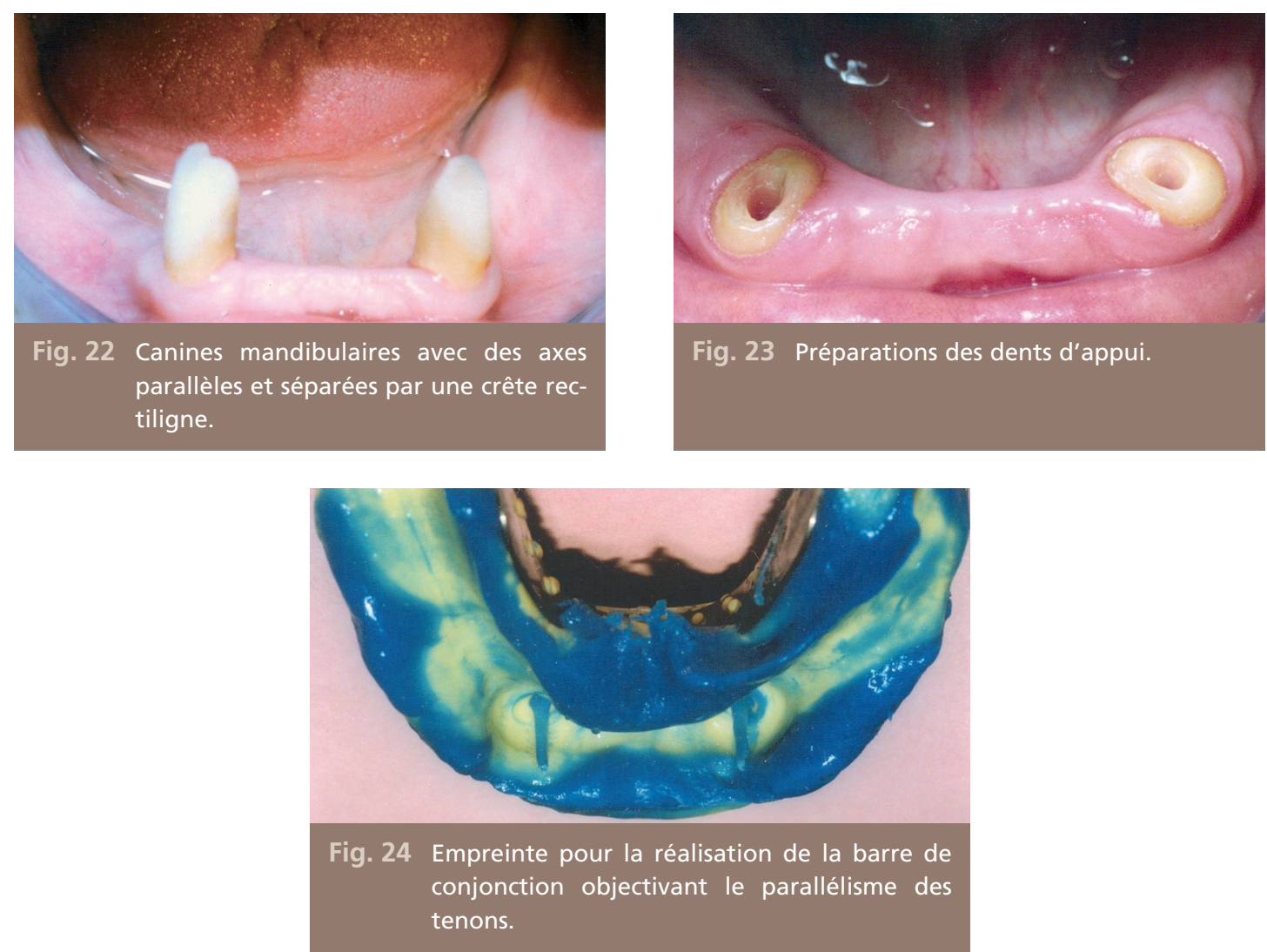

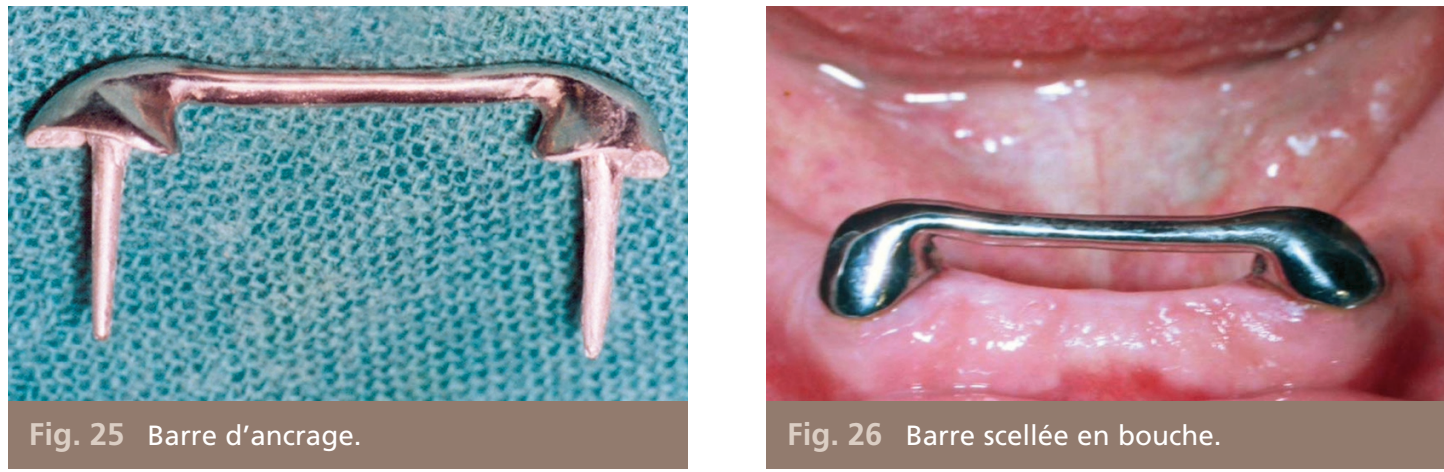

Fig. 26 Barre scellée en bouche.

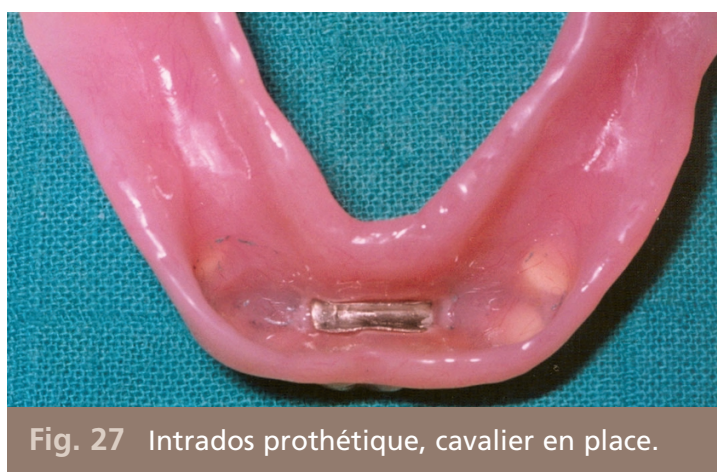

L'exploitation des racines permet de gérer au mieux le passage à l'édentation complète avec une meilleure approche psychologique grâce à la préservation de la proprioception et du capital osseux ainsi que l'augmentation de la rétention.

\section{Prothèse mandibulaire supra-implantaire}

En cas d'absence des racines dentaires, l'alternative implantaire demeure envisageable $s^{\prime} i l$ n'y a pas de contre-indication spécifique.

En effet, l'implant n'est plus à l'heure actuelle une technique novatrice expérimentale; I'ostéo-intégration donne des résultats satisfaisants et permet de retrouver l'équivalent d'une racine sous-prothétique.

\section{> Bilan préopératoire}

Toutes les thérapeutiques implantaires nécessitent une étude et une analyse préalable rigoureuse, celle-ci comprend :

- la qualité de la muqueuse buccale, la zone d'émergence de l'implant doit être située dans une bande de gencive kératinisée. Cette dernière joue un rôle indiscutable dans la stabilisation de l'environnement parodontal et conditionne la qualité du joint gingivoimplantaire ;

- l'étude des modèles sur articulateur permet d'évaluer si l'espace interarcade est suffisant pour placer les implants et la prothèse susjacente, ainsi que les rapports entre le maxillaire et la mandibule (normo-, rétro- ou prognathie) ;

- un bilan radiographique sert à apprécier le support osseux et les structures anatomiques 
(nerf dentaire mandibulaire, nerf mentonnier) situés dans les zones susceptibles de recevoir les implants ;

Les guides radiologique et chirurgical destinés à l'implantologie orale présentent essentiellement deux intérêts :

- les examens tomodensitométriques, ils permettent de visualiser les émergences souhaitées des implants, ainsi que des axes prothétiques. Des coupes de reconstruction sont faites dans l'axe matérialisé (sur le guide radiologique par de la gutta ou des tiges de titane) ;

- simplifier l'acte du chirurgien: les repères radiologiques pouvant se superposer aux repères des axes et émergences représentés sur le guide permettent alors une mise en place optimale des implants en harmonie avec le cadre fonctionnel. Un implant situé dans l'axe de la dent prothétique reçoit des forces favorables à l'ostéo-intégration avec un minimum de contraintes sur les vis de solidarisation $[21,22]$.

\section{$>$ Impératifs}

La pérennité du traitement en PACSI (Prothèse Adjointe Complète Supra-Implantaire) est directement subordonnée à la conception et à l'élaboration d'une prothèse amovible complète classique parfaitement équilibrée et intégrée ainsi qu'à l'emplacement des implants et au choix des éléments de rétention complémentaires qui offrent un jeu axial (translation verticale) et/ou un jeu angulaire (rotation distale) pour prendre en compte la différence de dépressibilité tissulaire existant entre la fibromuqueuse de la crête édentée et l'implant [23]. Ainsi, la réussite d'une réhabilitation par PACSI dépend d'un certain nombre de paramètres :

- la qualité des empreintes: même si des moyens de rétention mécaniques seront mis en place, la réalisation d'une empreinte précise reste indispensable à l'obtention d'un équilibre prothétique en majorant la sustentation par une exploitation maximale de la surface d'appui, ce qui évite la surcharge des implants et donc assure leur pérennité ;

- l'enregistrement précis du rapport intermaxillaire et l'établissement d'un équilibre occlusal inscrit dans une occlusion bilatéralement équilibrée sont les seuls garants de la préservation des surfaces d'appui et sont favorables à l'obtention et au maintien de l'ostéo-intégration des implants [24] ;

- le nombre d'implants : à la mandibule, deux à quatre implants peuvent être placés dans la symphyse, en général deux suffisent pour assurer significativement et de manière durable l'équilibre d'une prothèse amovible $[25,26]$.

Pour les cas de barre, l'augmentation du nombre d'implants symphysaires au-delà de deux permet d'accroître la rétention, de préserver l'ostéo-intégration et d'obtenir une meilleure efficacité masticatoire [27].

Une prothèse amovible retenue par deux implants symphysaires représente le traitement minimal de l'édentement total mandibulaire (consensus de Mac Gill) [28].

- La situation des implants est symphysaire entre les deux foramens mentonniers.

- Le système de rétention peut être de type barre ou bouton-pression.

Habituellement, les attachements axiaux sur racines naturelles se situent au niveau des canines, la position idéale pour les implants se situe au niveau des incisives latérales.

Cette situation antérieure permet de caler la prothèse lors de l'incision, et de ne garder qu'un seul mouvement d'enfoncement postérieur, plutôt qu'un double mouvement si les attachements sont situés au niveau des canines [16]. 
- Le mode de connexion : il doit permettre une rétention complémentaire d'une prothèse amovible complète bien conçue. La sustentation et la stabilisation sont assurées par les surfaces d'appui ostéo-muqueuses [25].

L'espace prothétique minimum nécessaire au niveau du site implantaire envisagé est pour la plupart des systèmes d'attachements préconisés de $7 \mathrm{~mm}$ de hauteur et de $5 \mathrm{~mm}$ de largeur. Le respect de ce volume d'encombrement permet de concilier la solidité et un volume idéal de PACSI [23].

\section{> Le choix du système d'attachements PACSI selon la situation clinique}

- Une crête antérieure rectiligne indique la réalisation d'une barre de conjonction. Une barre curviligne reliant deux implants crée un bras de levier défavorable. La diminution de la courbure impose un surcontour prothétique lingual qui entraîne un handicap fonctionnel majeur [26]. L'augmentation du nombre d'implants permet de segmenter en plusieurs parties rectilignes favorables à la réalisation d'une barre de conjonction.

- Une résorption osseuse importante préconise l'augmentation du nombre d'implants réunis par une barre rigidifiant le système implantaire. Le rapport hauteur prothétique/hauteur implantaire défavorable contre-indique le choix d'attachements non solidarisés.

- Un espace prothétique limité évalué à l'aide de clés ou de guides de positionnement indique le choix d'attachements axiaux de taille réduite.

- Dans le cas d'aparallélisme des piliers implantaires, si une faible divergence entre les attachements axiaux ne compromet pas l'insertion prothétique, elle provoque néanmoins une accélération de l'usure du dispositif de rétention. Les systèmes axiaux à rattrapage d'angulation constitués d'une base transvis- sée dans l'axe de l'implant avec une partie mâle compensent l'aparallélisme.

- Une distance inter-implantaire entre $10 \mathrm{~mm}$ et $15 \mathrm{~mm}$ est nécessaire pour mettre un cavalier rétentif sur barre sans déformation.

- Une arcade mandibulaire étroite est en faveur des attachements axiaux en limitant le nombre d'implants.

- La mise en charge immédiate chez l'édenté complet représente une indication de barre de conjonction, en réponse à l'impératif de stabilité primaire des implants nécessaire à l'ostéo-intégration [13.]

\section{> La maintenance}

La maintenance individuelle est associée à une maintenance professionnelle, cette dernière consiste en un contrôle de l'équilibre implanto-dento-prothétique :

- au niveau osseux par un contrôle radiologique vérifiant l'absence de "cratérisations» autour des implants ;

- au niveau gingival : par une appréciation du contrôle de plaque, de la motivation du patient et de l'état de santé des tissus périimplantaires. Ceci permet d'intercepter toute altération pouvant compromettre l'ostéointégration ;

- au niveau occlusal : le contrôle de l'occlusion pour éviter toute surcharge au niveau des implants.

Un nettoyage professionnel comprenant des séances de détartrage-surfaçage suivi d'un polissage soigneux est réalisé.

Les séances de maintenance se font à un mois après la pose de la prothèse, puis tous les 6 mois pendant 2 ans puis une fois par an [29]. En cas de diminution de la rétention initiale, on procède au changement des pièces responsables de la rétention (remplacement des anneaux élastiques, ou activation). 


\section{Cas clinique $n^{\circ} 4$}

Une patiente de 57 ans s'est présentée pour la réalisation d'une nouvelle prothèse complète bimaxillaire, insatisfaite de son ancienne prothèse conventionnelle psychiquement inacceptable et dont la rétention est insuffisante par suite d'une résorption de la crête résiduelle. La thérapeutique implantaire a été proposée à la patiente.

L'objectif du traitement est la réalisation $d$ 'une prothèse complète bimaxillaire avec au niveau mandibulaire un système d'attachement type bouton-pression au niveau des quatre implants mandibulaires.

Tout d'abord, le projet prothétique est validé : par la réalisation d'un montage des dents artificielles sur des moulages montés sur articulateur.

La patiente et le praticien ont ainsi validés chacun de ces objectifs et ont eu à disposition la position, la dimension, la forme et la couleur des dents artificielles (fig. 28, 29) [30].

Un bilan pré-implantaire a été fait et quatre implants ont été mis en place.

Une radiographie panoramique de contrôle a permis après la mise en place des implants de vérifier le positionnement et les axes des implants (fig. 30).

Des attachements $\mathrm{O}^{\prime}$ Ring ${ }^{\circledR}$ ont été prévus : conçus pour une utilisation large dans n'importe quel cas en prothèse adjointe, ils consistent en une patrice en titane vissée sur la fixture, en une matrice rouge servant de transfert et en une bague $\mathrm{O}^{\prime} \mathrm{Ring}^{\circledR}{ }^{\circledR}$ blanche interchangeable insérée dans l'étui métallique [12].
Les vis de cicatrisation ont été enlevées et les parties mâles des attachements ont été vissées sur les implants (fig. $\mathbf{3 1}$ ).

Par la suite, les parties femelles sont repositionnées sur les parties mâles (fig. 32).

L'intrados prothétique en regard des parties mâles a été évidé dans le but de ménager l'espace des parties femelles ainsi que de la résine qui assure la solidarisation de ces dernières à l'intrados de la prothèse mandibulaire (fig. 33).

Un silicone de faible viscosité permet de détecter les zones de compression et par conséquent de guider les retouches préalables à la solidarisation des parties femelles à I'intrados (fig. 34).

Après élimination de toute compression, une petite quantité de résine auto-polymérisable est placée au niveau de l'intrados à l'emplacement exact des parties femelles dans la prothèse et suivie immédiatement de la mise en place de la prothèse mandibulaire et son maintien sous pression occlusale jusqu'à polymérisation de la résine. De la cire a été préalablement placée autour de la pièce mâle afin d'éviter la fusion de la résine sous les attachements, ce qui rendrait la désinsertion impossible.

La prothèse est ensuite retirée (fig. 35). L'occlusion est parfaitement contrôlée. La rétention nettement améliorée a donc facilité l'intégration psychologique de la prothèse témoignant du succès thérapeutique (fig. 36 ). 


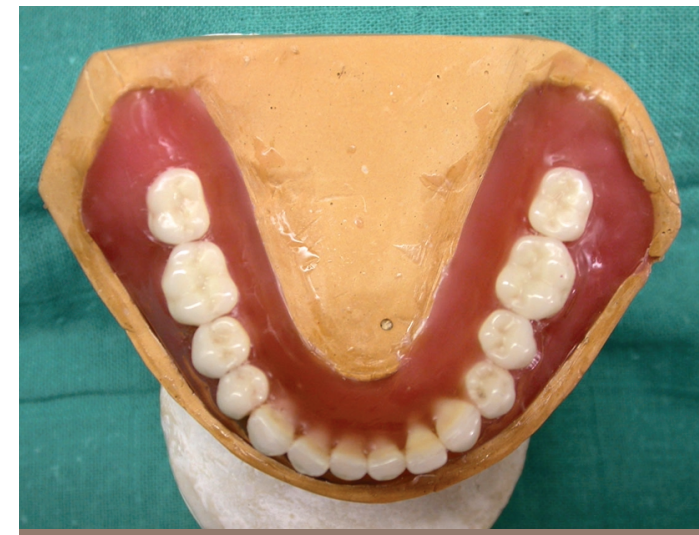

Fig. 28 Vue occlusale de la prothèse mandibulaire.

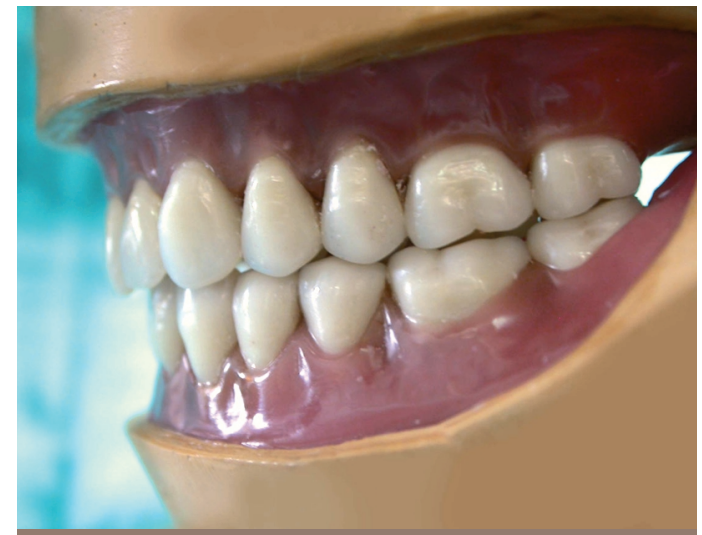

Fig. 29 Vue latérale de la prothèse bimaxillaire élaborée selon les données diagnostiques.

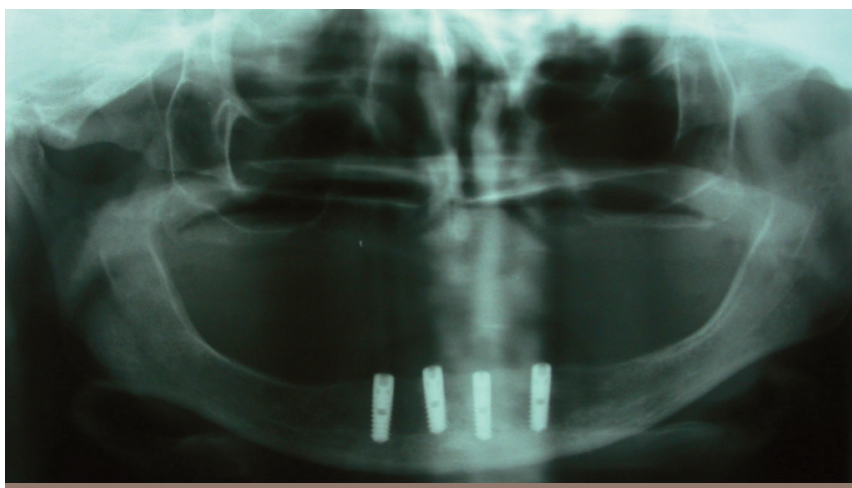

Fig. 30 Radiographie panoramique de contrôle après mise en place des implants.

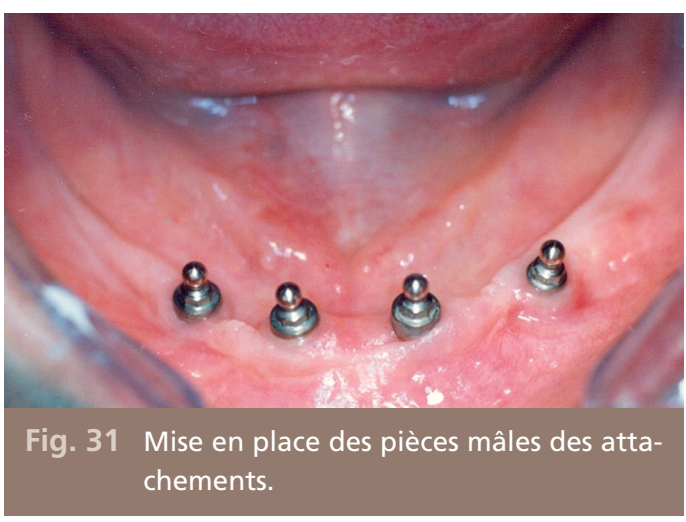

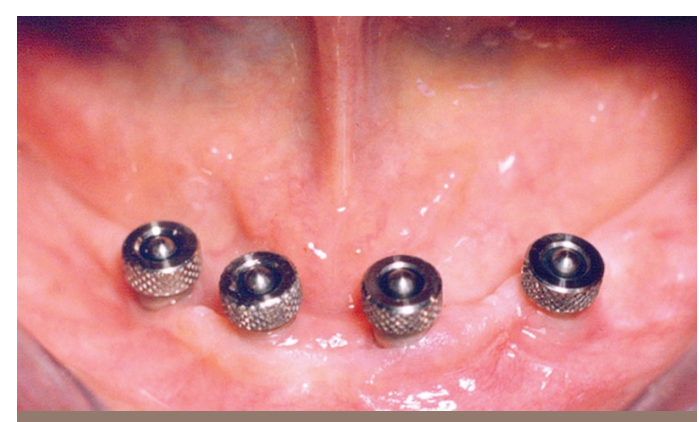

Fig. 32 Placement des pièces femelles. 

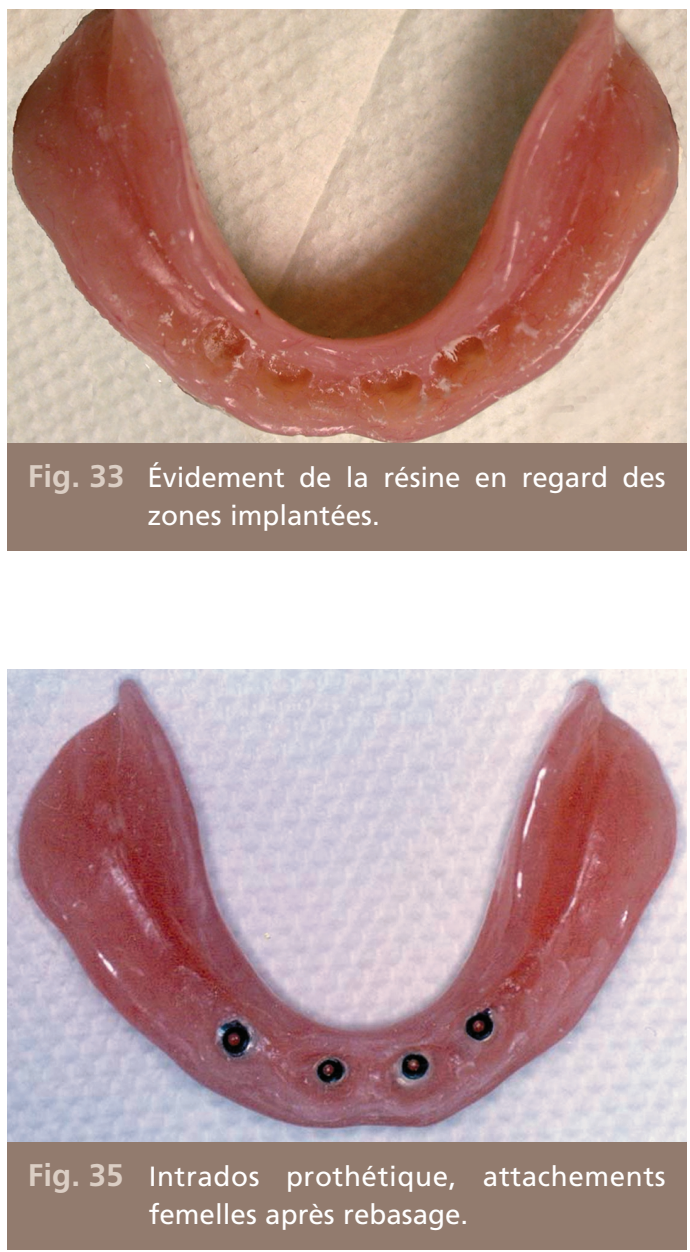

\section{Conclusion}

Les différentes étapes d'élaboration d'une prothèse complète mandibulaire interviennent simultanément et conjointement pour aboutir à un équilibre prothétique.

L'utilisation des différentes techniques visant à améliorer la stabilité et la rétention de nos prothèses que ce soit par le biais d'attachements implantaires ou radiculaires ne dispense pas le praticien de réaliser rigoureusement les étapes classiques de la prothèse complète. II

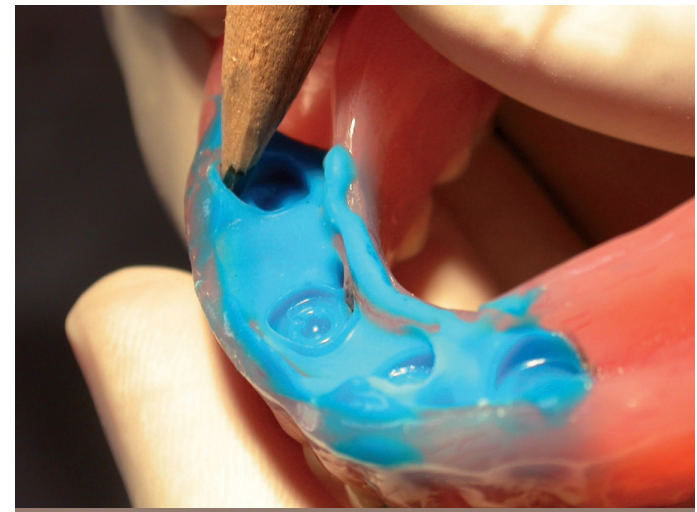

Fig. 34 L'espacement contrôlé avec un silicone fluide.

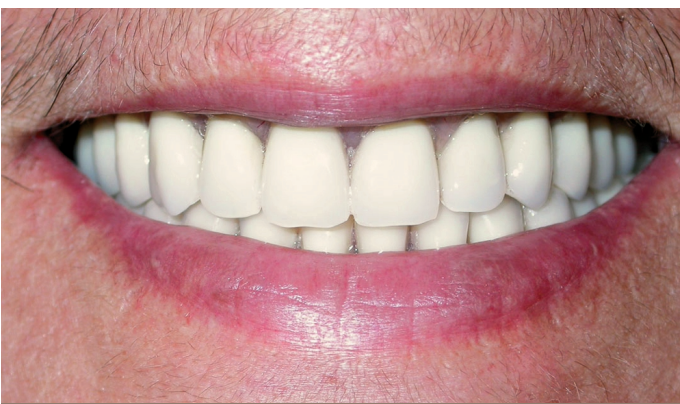

Fig. 36 Sourire de satisfaction de la patiente. convient de les effectuer d'une manière précise afin de pérenniser les supports de rétention et d'éviter un éventuel échec thérapeutique.

Les séances de maintenance permettent de réadapter la prothèse en cas de résorption osseuse, de réactiver les éléments rétentifs en cas de perte de rétention et enfin de contrôler et d'intercepter à temps une quelconque complication au niveau des piliers prothétiques. 
Bibliographie

1. Gibert $Y$, Soulet $H$, Blandin $\mathrm{M}$.

Phénomènes rétentifs en prothèse adjointe complète.

Encycl Méd Chir (Paris, France), Odontologie, 23325 B05, 4-1987, 6p.

2. Schoendorff $\mathrm{R}$, Millet $\mathrm{C}$. Rétention en prothèse complète. Encycl Méd Chir (Paris-France), Stomatologie-Odontologie II, 23-325-B-05, 1995, 6p.

3. Martin JP, Citterio $\mathrm{H}$, Coeuriot JL. Amélioration de la stabilité dynamique des prothèses adjointes totales ; proposition pour une conduite anatomo-physiologique de la restauration prothétique. Rev Odonto Stomatol 1993;22(5).

4. Samoian R.

Apport des techniques piézographiques en prothèse adjointe totale. Act Odonto-Stomatol 1992;177:157-177.

5. Klein $\mathrm{P}$

Propos sur la piézographie. Inf Dent 1972;54(33):3115-3119.

6. Klein P.

La piézographie : modelage dynamique, ou volume prothétique. Act Odonto-Stomatol 1974;106:266-277.

7. Nabid A, Klein P. Méthodologie du moulage de l'espace prothétique mandibulaire gérodontologique :
Technique phonétique. Odontologia 1984;6:31-44.

8. Laveau $\mathrm{A}$.

Espace prothétique mandibulaire

en prothèse dentaire adjointe totale et piézographie. Inf Dent 1986;30:2875-2885.

9. Amzalag $\mathrm{G}$, Batarec $\mathrm{E}$, Schoendorff R, Buch D, Assemat-Tessandier $\mathrm{X}$. Prothèses supraradiculaires "overdentures".

Paris : Éditions CdP 1988:3-10.

10. Dabadie M.

Quand I'implant remplace avantageusement

les racines

sous-prothétiques.

Les cahiers de l'ADF 1999;6:16-21.

11. Budz Jorgensen $E$, Clavel.R. La prothèse totale : théorie, pratique et aspects médicaux. Paris : Éditions Masson 1995:125-130.

12. Manglavite $R$, Nesler PL. Rétention et usure des attachements de précision pour prothèse sur implants ostéo-intégrés. Cah Prothèse 1992;78:79-89.

13. Fromentin $O$.

Critères de choix des systèmes d'attachements en prothèse amovible complète supra-implantaire (PACSI). Réalités cliniques 2003;14(2):161-171.

14. Martini LF.

Attachements

et prothèse composite.

Paris : Masson

1992;67-74:89-93.
15. Batarec E, Buch D. Abrégé de prothèse adjointe partielle. Paris : Éditions Masson 1989:123-131.

16. Assémat-Tessandier $X$. La prothèse supra-implantaire mandibulaire : les boutons-pression. Implant 2000;6(2):114-122.

17. Unger F, Hoornaert $A$, Estrabaud $\mathrm{Y}$. Indication et mise en œuvre attachements axiaux supra-radiculaires. Réalités Cliniques 1998;9(4):513-523.

18. Postaire M, Naser B. Prothèse complète mandibulaire supra-implantaire : un protocole de réalisation. Cah prothèse 1996;95:7-16.

19. Blanc J, Blum JY, Isnard L. Prothèses totales amovibles de recouvrement sur implants. Mode de rétention associant barre de connexion et silicone. Cah Prothèse 2002;117:27-34.

20. Rignon-Bret JM, Pompignoli $\mathrm{M}$.

La barre de rétention contra-muqueuse en prothèse complète mandibulaire.

Cah Prothèse 1989;68:57-70.

21. Augthun $\mathrm{M}$, Bieniek $\mathrm{K}$, Spiekermann $\mathrm{H}$. Le plan de traitement des restaurations prothétiques implanto-portées chez l'édenté total. Cah prothèse 1992;78:57-63. 
22. Ré JP, Orthlieb JD, Glise JM, Laurent $\mathrm{M}$.

Optimisation

du positionnement

des implants

à la mandibule.

Cah Prothèse 2004;128:73-79.

23. Rignon-Bret $C$, Descamp $F$, Bernaudin $\mathrm{E}$, Bloch $\mathrm{M}$, Hadida A.

Stratégie de traitement en prothèse amovible complète supra-implantaire mandibulaire.

Réalités cliniques 2003;14(2):141-159.

24. Chiche F, Guez G. Actualisation des concepts occlusaux en implantologie. Cah Prothèse 2000;112:83-96.

25. Postaire $M$, Rignon-Bret $C$, Daas M, Renouard F,
Rignon-Bret JM.

Conception des prothèses

amovibles complètes

supra-implantaires

mandibulaire.

Réalités cliniques

2003;14(2):199-212.

26. Rignon-Bret $C$,

Rignon-Bret JM.

Prothèse amovible complète

supra-implantaire.

In: Prothèse amovible

complète.

Prothèse immédiate.

Prothèses supraradiculaire

et implantaire.

Rueil-Malmaison :

Édition CdP 2002:187-219.

27. Bluche $\mathrm{L}$, Armand $\mathrm{S}$.

PACSI

(prothèses amovibles

complètes sur implants).
Aide à la conception

des barres

à la mandibule.

Cah Prothèse 2003;123:7-15.

28. Veyrune JL, Lassauy $C$, Cornet A, Lesher J.

Apport des implants dans le traitement de l'édentement total mandibulaire.

Cah Prothèse 2005;130:59-66.

29. Picard B, Tavernier B, Hary F, Bussac G.

Prothèse implantaire.

Paris : Édition CdP 2000:67-71.

30. Mariani P, Stephan G.

Implants

et édentement total,

quel protocole

thérapeutique?

Cah Prothèse

2002;120:27-36.

\section{SUMMARY}

\section{Complete mandibular denture: stability and retention}

Leila FAJRI,

Faiza BENFDIL,

Bouabid EL MOHTARIM,

Wafaa EL WADY,

Ahmed ABDEDINE

$$
\begin{aligned}
& \text { Keywords } \\
& \text { - complete mandibular } \\
& \text { denture } \\
& \text { - prosthetic balance } \\
& \text { - attachments } \\
& \text { - overdenture supported } \\
& \text { by natural teeth } \\
& \text { - the implant-supported } \\
& \text { overdenture }
\end{aligned}
$$

The retention problem is one of the most frequent patient's complaints in complete mandibular dentures.

Stability and retention of removable complete mandibular denture requires on the one hand to reproduce supporting surfaces and on the other hand to adopt the right occlusal concept.

Responding to diverse clinical situations and economical conditions, many solutions to the problem of low edentulism can be envisaged.

The neutral zone impression technique is useful when providing mandibular complete dentures. Its use can overcome some of the denture stability difficulties caused in some patients by a more powerful oral musculature, or in patients who have poor or altered neuromuscular control. Over denture supported by natural teeth is widely used in clinical practice and should be executed whenever the clinical conditions suggest it. The greater retention and stability of over denture in comparison with complete denture greatly improve the masticatory efficacy. The current development of implantology have proved that Implant-supported prostheses to be a great therapeutic value making easier the prosthesis integration and improving retention and patient's quality of life. 